\title{
Hidrodinâmica relativística: a representação de diversos fluidos em relatividade geral
}

\author{
Relativistic hydrodynamics: the representation of several fluids in general relativity
}

\author{
Rodrigo Francisco dos Santos ${ }^{1,2}$, Antônio Carlos Amaro de Faria Júnior ${ }^{3,4}$, Sérgio Costa Ulhoa ${ }^{*}[0$ \\ ${ }^{1}$ Universidade Federal Fluminense, Instituto de Física, Rio de Janeiro, RJ, Brasil. \\ ${ }^{2}$ Fundação Centro de Educação a Distância do Estado do Rio de Janeiro, Polo Nova Iguaçu, Nova Iguaçu, RJ, Brasil. \\ ${ }^{3}$ Universidade Tecnológica Federal do Paraná, Guarapuava, PR, Brasil. \\ ${ }^{4}$ Instituto de Estudos Avançados, São José dos Campos, SP, Brasil. \\ ${ }^{5}$ Universidade de Brasília, Instituto de Física, Brasilia, DF, Brasil
}

Recebido em 06 de Janeiro de 2019. Revisado em 08 de Julho de 2019. Aceito em 10 de Setembro de 2019

\begin{abstract}
O tensor de momento-energia é a entidade matemática que representa de forma unificada as fontes de momento e energia no formalismo covariante, tanto em espaços planos, como em espaços curvos. Em espaços curvos o tensor de momento-energia fica conectado a curvatura do espaço-tempo via equação de campo de Einstein. O tensor de momento-energia caracteriza os campos de matéria do sistema. Por sua vez as condições de energia estabelecidas por Hawking e Ellis classificam os diversos tipos de fluidos quanto a atratividade/repulsividade, a causalidade, interação com o vacuo e a positividade. Tambem abordamos a conservação do tensor de momento-energia via equação Tolemam-Openhaimer-Volkov(TOV), que é um importante formalismo para o estudo de estruturas e modelos estelares. Vamos estudar o tensor momento-energia nas suas versões isotrópicas e anisotrópicas, bem como a sua conservação e relação com a constante cosmológica.
\end{abstract}

Palavras-chave: Hidrodinâmica; Fluidos Relativísticos.

\begin{abstract}
The energy-momentum tensor is the mathematical entity that represents the sources of momentum and energy in a covariant formalism, both in flat and curved spaces. In curved spaces the energy-momentum tensor is connected tBo the space-time curvature via the Einstein field equation. The energy-momentum tensor characterizes the matter fields of the system. In turn, the energy conditions established by Hawking and Ellis classify the various types of fluids according to their attractiveness/repulsiveness, causality, interaction with the vacuum and positivity. We also address the conservation of the energy-momentum tensor via the Tolemam-Openhaimer-Volkov (TOV) equation, which is an important formalism for the study of stellar structures and models. We will study the energy-momentum tensor in its isotropic and anisotropic versions, as well as its conservation and relation to the cosmological constant.
\end{abstract}

Keywords: Hydrodynamics; Relativistic Fluids.

\section{Introdução}

Historicamente desde a Grécia antiga, que a humanindade tem interesse pelo estudo de fluidos [1]. Uma história muito popular e quase folclórica foi o famoso "Eureka" de Arquimedes após resolver um problema ligado ao empuxo, supostamente durante um banho de imersão.

O estudo dos fluidos e seu escoamento foi por séculos uma área de grande importância para o desenvolvimento da tecnologia, nomes como Stokes e Mach construiram conceitos matemáticos capazes de entender o movimento de fluidos, sendo extensíveis aos campos gravitacionais e eletromagnéticos [2].

O eletromagnetismo tratava os campos eletromagnéticos como fluidos, a própria idéia de corrente conservada, já continha em si a idéia de escoamento, onde o campo

*Endereço de correspondência: sc.ulhoa@gmail.com elétrico estaria associado ao fluxo por uma região determinada e o campo magnético associado aos vórtices em torno da corrente. As cargas seriam as fontes e os sorvedouros dos campos. Essa idéia foi incrementada com o advento da relatividade restrita, campos elétricos e magnéticos passaram a ter uma drescição unificada.

Em relatividade geral e algumas sub-áreas como cosmologia e astrofísica um dos principais debates é acerca do tipo de matéria, que está gerando o campo gravitacional. Nas equações de Einstein [3 a fonte do campo é a o tensor de momento-energia o tipo de matéria é um fluido caracterizado por sua equação de estado, que seria uma limitação imposta sobre o tensor de momento-energia. Contudo até o trabalho de Hawking-Ellis [6, 7. não havia qualquer limitação às equações de estado. Hawnking-Ellis introduziram as chamadas condições de energia que são imposições sobre o tensor de momento-energia quanto 
a propagação do fluido por ele descrito. Estudar essas condições de energia é hoje uma tarefa importante para pesquisadores, que trabalham com inflação [8], estrelas estranhas [9], energia escura, [5, 10 13, campos escalares [14], matéria de quark 15] e uma vasta quantidade de temas. Não existe limite para a violação das condições de energia, na verdade todas podem ser violadas, mas a violação de cada uma delas implica um tipo de fluido. O tensor de momento-energia mais simples que existe é o chamado tensor de poeira, que se trata de um fluido não interagente. Assim cada interação requer um termo a mais. A introdução de um termo proporcional à métrica implica energia do vácuo [5, 10], propriedades de anisotropia implicam uma quadrivelicdade com componente radial, porém sem quebra da simetria esférica, em geral esta é associada a introdução de campos eletromagnéticos 16. Todos estes tipos de tensores de fluido relativístico ideal, obedecem à conservação de momento e energia, derivada da simetria de Bianchi do tensor de Einstein. Essa simetria é importante no estudo de fluidos que compõem estrelas, sendo uma das mais ativas áreas da astrofísica atualmente 17.

Propriedades de superfluidos têm sido observada 18 em sistemas gravitacionais, o que levou ao surgimento de uma nova área de pesquisa que seria o estudo da estrutura causal da propagação de ondas em colóides, com o intuito de gerar analogias à estrutura causal em campos gravitacionais de objetos colapsados [19]. O trabalho está dividido da seguinte forma: Seção 2: Estudamos os campos eletromagnéticos como fluidos, explicando as relações com vórtices e escoamentos. Seção 3: Deduzimos o tensor de Maxewell, aprofundando as propriedades de fluidos e observando a equação da continuidade. Seção 4: Construímos o tensor de de momento-energia associado ao campo eletromagnético. Seção 5: O tensor de momento-energia é estudado como formalismo para descrever um fluido ideal, as restrições às equações de estado associadas às condições de energia são estabelecidas. Estudamos ainda a conservação do tensor de momento-energia para uma métrica genérica e para um objeto esférico. Seção 6: Estudamos um tensor de momento-energia com propriedade de anisotropia, estudamos as condições de energia associadas a esse tipo de fluido e demonstramos a conservação do tensor de momento-energia anisotrópico Seção 7: É construido um tensor de momento-energia associado a constante cosmológica e estudada a sua conservação.

\section{A eletrodinâmica como uma teoria de fluido}

Os experimentos originais do eletromagnetismo concebiam a corrente como um fluido de portadores de carga, que se propagava pelos condutores. A própria idéia de campos também trazia em seu cerne o conceito de fluido, sendo o caso do campo elétrico um fluido, o fluxo era calculado pela lei de Gauss

$$
\vec{\nabla} \cdot \vec{E}=\frac{\rho}{\epsilon_{0}}
$$

e carga $q=\int_{V} \rho d V$. Vórtices associados a esse fluido são descritos pela lei de Faraday

$$
\vec{\nabla} \times \vec{E}=-\partial_{t} \vec{B}
$$

onde o campo magnético é associado à propriedade de circulação do fluido. Além disso divergente do campo magnético é nulo

$$
\vec{\nabla} \cdot \vec{B}=0,
$$

que mostra a inexistência de fontes magnéticas (monopolos), completando a simetria entre os campos, temos a lei de Ampere-Maxwell

$$
\vec{\nabla} \times \vec{B}=\mu_{0} \vec{J}+\mu_{0} \epsilon_{0} \partial_{t} \vec{E},
$$

sendo $\mu_{0} \epsilon_{0} \partial_{t} \vec{E}$ a corrente de deslocamento descoberta por Maxwell. Na ausência de fontes $q=0, \vec{j}=0$, temos

$$
\begin{gathered}
\vec{\nabla} \cdot \vec{E}=0, \\
\vec{\nabla} \times \vec{E}=-\partial_{t} \vec{B}, \\
\vec{\nabla} \cdot \vec{B}=0, \\
\vec{\nabla} \times \vec{B}=\mu_{0} \epsilon_{0} \partial_{t} \vec{E} .
\end{gathered}
$$

Portanto o vórtice de um campo elétrico implica na variação temporal do campo magnético, assim como o vórtice magnético implica na variação temporal do campo elétrico. Aplicando o rotacional em (8), temos:

$$
\nabla^{2} \vec{B}-\frac{1}{c^{2}} \partial_{t}^{2} \vec{B}=0
$$

logo vemos, a equação de onda para o campo magnético, podemos da mesma forma deduzir para o campo elétrico aplicando o rotacional na equação (6)

$$
\nabla^{2} \vec{E}-\frac{1}{c^{2}} \partial_{t}^{2} \vec{E}=0
$$

Embora os campos sejam similares a fluidos, a onda eletromagnética não se propaga em nenhum meio, essa foi uma das maiores revoluções da ciência, pois colocou fim a ideia do éter luminífero, que mais tarde a experiência de Michelson-Morley demonstrou não existir.

\section{O Tensor de Maxwell}

Podemos definir os campos auxiliares, chamados potenciais eletromagnéticos $\Phi$ e $\vec{A}$. Em seguida escrevemos os campos elétrico e magnético em termos dos potenciais,

$$
\begin{gathered}
\vec{E}=-\vec{\nabla} \Phi-\frac{1}{c} \partial_{t} \vec{A}, \\
\vec{B}=\vec{\nabla} \times \vec{A} .
\end{gathered}
$$


Esses potenciais tem um papel importante por exemplo no efeito Aharonov-Bohm. Podemos unificar os campos e contruir o formalismo tensorial. Definimos então o quadrivetor potencial

$$
A^{\mu}=\left(A^{0}, A^{1}, A^{2}, A^{3}\right)
$$

onde $A^{0}=\Phi$, e $A^{1}, A^{2}, A^{3}$, são as componentes do vetor potencial, unificando tambem as derivadas $\partial_{\mu} \equiv \frac{\partial}{\partial x^{\mu}}=$ $\left(\frac{1}{c} \partial_{t}, \vec{\nabla}\right)$. Devemos notar que os índices subscritos e sobrescritos se referem à propriedades de transformação de coordenadas, tais propriedades são conhecidas como contravariância e covariância respectivamente. A relação entre essas quantidades é dada pelo tensor métrico $g_{\mu \nu}$ que é definido pelo produto escalar da base de um determinado espaço. Podemos definir o tensor de Maxwell

$$
F^{\mu \nu}=\partial^{\mu} A^{\nu}-\partial^{\nu} A^{\mu}
$$

Explicitamente escrevemos, usando $i=1,2,3$ :

$$
\begin{gathered}
F^{0 i}=E^{i}=-\partial^{0} A^{i}-\partial^{i} A^{0}, \\
F^{i j}=\partial^{i} A^{j}-\partial^{j} A^{i}=B_{k} \epsilon^{i j k} .
\end{gathered}
$$

Escrevemos finalmente a matriz associada ao tensor Maxwell

$$
F^{\mu \nu}=\left[\begin{array}{rrcc}
0 & E^{1} & E^{2} & E^{3} \\
-E^{1} & 0 & B^{3} & B^{2} \\
-E^{2} & -B^{3} & 0 & B^{1} \\
-E^{3} & -B^{2} & -B^{1} & 0
\end{array}\right]
$$

Conhecendo a equação da continuidade

$$
\vec{\nabla} \cdot \vec{j}+c \partial_{t} \rho=0
$$

onde $\vec{j}, \rho$ são a densidade de corrente vetorial e a carga, podemos generalizar essa corrente (18) usando a equação da continuidade 18

$$
\partial_{\mu} J^{\mu}=0
$$

temos então a quadridivergencia da quadri-corrente $J^{\mu}=$ $(\rho, \vec{j})$. Se agimos com o operador derivada no tensor de Maxwell

$$
\partial_{\mu} F^{\mu \nu}=\mu_{0} J^{\nu}
$$

de forma mais explicita, se agimos $\partial_{i} F^{0 i}=\partial_{i} E^{i}=$ $\nabla A^{0}=\frac{\rho}{\epsilon_{0}}$, e ainda, $\partial_{i} F^{i j}=\mu_{0} j^{j}$ onde $J^{j}=\vec{j}$.

\section{Tensor de momento-energia: fluido relativistico}

Vamos definir a ação seguindo 3 .

$$
S=\int \xi\left(q, \frac{\partial q}{\partial x^{i}}\right) d V d t=\frac{1}{c} \int \xi d \Omega
$$

onde, $q_{, i} \equiv \frac{\partial q}{\partial x_{i}}$ são variáveis generalizadas.
Minimizando a ação (21)

$$
\begin{gathered}
\delta S=\frac{1}{c} \int\left(\frac{\partial \xi}{\partial q} \delta q+\frac{\partial \xi}{\partial q_{, i}} \delta q_{, i}\right) d \Omega \\
=\frac{1}{c} \int\left[\frac{\partial \xi}{\partial q} \delta q \frac{\partial}{\partial x^{i}}\left(\frac{\partial \xi}{\partial q_{, i}}\right)-(\delta q) \frac{\partial}{\partial x^{i}} \frac{\partial \xi}{\partial q_{, i}}\right] d \Omega=0
\end{gathered}
$$

o segundo termo se anula sobre a integração em todo o espaço. Podemos então escrever a equação do movimento como

$$
\frac{\partial}{\partial x^{i}} \frac{\partial \xi}{\partial q_{i}}-\frac{\partial \xi}{\partial q}=0
$$

aqui assumimos a soma sobre indices repetidos. Seguindo agora um procedimento similiar ao usado para verificar a conservação da energia

$$
\frac{\partial \xi}{\partial x^{i}}=\frac{\partial \xi}{\partial q} \frac{\partial q}{\partial x^{i}}+\frac{\partial \xi}{\partial q_{, k}} \frac{\partial q_{, k}}{\partial x^{i}},
$$

substituindo na equação de movimento , considerando que $q_{, k, i}=q_{, i, k}$, temos:

$$
\frac{\partial \xi}{\partial x^{i}}=\frac{\partial}{\partial x^{k}}\left(\frac{\partial \xi}{\partial q_{, k}}\right) q_{, i}+\frac{\partial \xi}{\partial q_{, k}} \frac{\partial q_{, i}}{\partial x^{k}}=\frac{\partial}{\partial x^{k}}\left(q_{, i} \frac{\partial \xi}{\partial q_{, k}}\right)
$$

usando a seguinte propriedade

$$
\frac{\partial \xi}{\partial x^{i}}=\delta_{i}^{k} \frac{\partial \xi}{\partial x^{k}} .
$$

O tensor de momento-energia em termos das variáveis canônica é:

$$
T_{i}^{k}=q_{, i} \frac{\partial \xi}{\partial q_{, i}}-\delta_{i}^{k} \xi
$$

Seguindo esta forma do tensor de momento-energia, o mais simples $3,5,10,11$

$$
T^{\mu \nu}=U^{\mu} P^{\nu},
$$

sendo $U^{\mu}=c \sqrt{1-\frac{v^{2}}{c^{2}}}\left[1 \cdot \frac{v^{\alpha}}{c}\right]$ a quadri-velocidade de um observador em movimento junto com o fluido e $P^{\mu}=$ $m_{0} c U^{\mu}$ o quadri-momentum. Usualmente especificamos as componentes do tensor de momento-energia também definimos nominalmente temos a normalização

$$
g_{\mu \nu} U^{\mu} U^{\nu}=1,
$$

a densidade de energia

$$
T^{00}=\rho,
$$

o fluxo de energia

$$
T^{i 0}=c P^{i}
$$

o tensor das tensões

$$
T^{i j}=u^{i} p^{i},
$$

aqui índices latinos limitam se a $i, j=1,2,3$ e índices gregos a $\mu, \nu=0,1,2,3$. O tensor de momento-energia é uma matriz simétrica, ou seja

$$
T^{\mu \nu}=T^{\nu \mu}
$$


Relacionando o tensor de momento-energia energia momentum a estrutura cinemática da relatividade especial , temos

$$
T^{\mu \nu}=m_{0} c \sqrt{1-\frac{v^{2}}{c^{2}}} U^{\mu} U^{\nu} .
$$

A conservação do tensor de energia momentum de poeira tem uma consequência fundamental,

$$
\partial_{\mu} T^{\mu \nu}=m_{0} c^{2}\left(\partial_{\mu} U^{\mu}\right) U^{\nu}=0 .
$$

Podemos definir a quadri-força como

$$
f^{\mu}=\partial_{t}\left(m U^{\mu}\right) \text {. }
$$

A força de Lorentz é também um quadri-vetor

$$
f^{\nu}=\frac{q}{c} U_{\mu} F^{\mu \nu}
$$

pela lei de Newton chegamos a

$$
m_{0} c \partial_{t} U^{\mu}=\frac{q}{c} U_{\mu} F^{\mu \nu},
$$

substituindo (38) em (35), temos

$$
\partial_{\mu} T^{\mu \nu}=\frac{q}{c} U_{\mu} F^{\mu \nu},
$$

a quadri-corrente pode ser escrita como $J^{\mu}=q U^{\mu}$, ficamos então:

$$
\partial_{\mu} T^{\mu \nu}=\frac{1}{c} J_{\mu} F^{\mu \nu}
$$

pela equação 20 chegamos que a

$$
\partial_{\mu} T^{\mu \nu}=\frac{\mu_{0}}{c}\left(\partial^{\alpha} F_{\alpha \mu}\right) F^{\mu \nu}
$$

usando as propriedades da derivada do produto

$$
\begin{aligned}
& \partial^{\alpha}\left(F_{\alpha \mu} F^{\mu \nu}\right)=\left(\partial^{\alpha} F_{\alpha \mu}\right) F^{\mu \nu}+F_{\alpha \mu} \partial^{\alpha} F^{\mu \nu} \\
& \partial^{\alpha}\left(F_{\alpha \mu} F^{\mu \nu}\right)-F_{\alpha \mu} \partial^{\alpha} F^{\mu \nu}=\left(\partial^{\alpha} F_{\alpha \mu}\right) F^{\mu \nu}
\end{aligned}
$$

e evocando a propriedade de simetria

$$
\begin{gathered}
\left(\partial^{\alpha} F_{\alpha \mu}\right) F^{\mu \nu}=\left[\partial^{\alpha}\left(F^{\mu \nu} F_{\alpha \mu}\right)-F_{\alpha \mu} \partial^{\alpha} F^{\mu \nu}\right] \\
F_{\alpha \mu} \partial^{\alpha} F^{\mu \nu}=\frac{1}{2}\left(F_{\alpha \mu} \partial^{\alpha} F^{\mu \nu}+F_{\mu \alpha} \partial^{\mu} F \nu \alpha\right) \\
=\frac{1}{2} F_{\alpha \mu}\left(\partial^{\alpha} F^{\alpha \mu}+\partial^{\mu} F^{\alpha \mu}\right)=-\frac{1}{2} F_{\alpha \mu} \partial^{\alpha} F^{\mu \alpha} \\
=-\frac{1}{4} \partial^{\nu}\left(F_{\alpha \mu} F^{\mu \alpha}\right),
\end{gathered}
$$

temos

$$
\frac{1}{c} F^{\mu \nu} J_{\mu}=\frac{1}{4}\left[\partial\left(F^{\alpha \mu} F_{\alpha \mu}\right)-\frac{1}{4} \partial^{\nu}\left(F_{\mu \alpha} F^{\mu \alpha}\right)\right]
$$

$\log \mathrm{O}$

$$
\partial_{\mu} T^{\mu \nu}=\frac{1}{4 \pi} \partial_{\alpha}\left(F^{\nu \mu} F_{\mu}^{\alpha}-\frac{1}{4} g^{\nu \alpha} F_{\mu \rho} F^{\mu \rho}\right) .
$$

Finalmente o tensor de momento-energia para o campo eletromagnético é

$$
T_{e m}^{\nu \alpha}=\frac{1}{4 \pi}\left(-F^{\nu \mu} F_{\mu}^{\alpha}-\frac{1}{4} g^{\nu \alpha} F_{\mu \rho} F^{\mu \rho}\right),
$$

operando com $g_{\nu \alpha}$, verificamos facilmente que o traço desse tensor eletromagnético é nulo. Matricialmente escrevemos o tensor de momento-energia como

$$
T^{\mu \nu}=\left[\begin{array}{cccc}
T^{00} & T^{01} & T^{02} & T^{03} \\
T^{10} & T^{11} & T^{12} & T^{13} \\
T^{20} & T^{21} & T^{22} & T^{23} \\
T^{30} & T^{31} & T^{32} & T^{33}
\end{array}\right] .
$$

Sabendo da propriedade de anti-simetria do tensor de Maxwell $F^{\mu \nu}=-F^{\nu \mu}$, facilmente verificavel em (17), aplicando essa mesma propriedade em (48) notamos que $T^{\mu \nu}=T^{\nu \mu}$. As componentes $T^{i j} \operatorname{com} i, j=1,2,3$ formam o chamado tensor das tensões. Já as componentes $T^{0 i}$

$$
T_{e m}^{0 i}=-\frac{1}{4 \pi} F^{0 \mu} F_{\mu}^{i}
$$

deotam o vetor de Poyting $\vec{S}=\vec{E} \times \vec{B}$ que é a densidade direcional de propagação de energia.

\section{Fluido isotrópico}

Inspirados em 27) definimos o tensor de momento-energia de um fluido ideal o mais geral possivel (sem anisotropia) $5,10,11$ como

$$
\begin{gathered}
T^{\mu \nu}=(\rho+p) U^{\mu} U^{\nu}+p g^{\mu \nu} \\
T^{\mu \nu}=\left[\begin{array}{rrcc}
\rho & 0 & 0 & 0 \\
0 & -p & 0 & 0 \\
0 & 0 & -p & 0 \\
0 & 0 & 0 & -p
\end{array}\right] .
\end{gathered}
$$

O traço do tensor de energia momento é

$$
T=\rho-3 p
$$

e a fonte de densidade de energia é dada por

$$
\rho=T^{\mu \nu} U_{\mu} U_{\nu}
$$

Podemos definir o projetor $P_{\mu \nu}=g_{\mu \nu}-U_{\mu} U_{\nu}$. Assim achamos a pressão

$$
-\frac{1}{3} P_{\mu \nu} T^{\mu \nu}=p
$$

O tensor de momento-energia pode ser reenscrito em termos do projetor como

$$
T^{\mu \nu}=\rho U^{\mu} U^{\nu}+p P^{\mu \nu} .
$$

Temos ainda os casos de um tensor de momento-energia $T^{\mu \nu}=\rho U^{\mu} U^{\nu}$, que é chamado de tensor de momentoenergia de poeira, neste caso temos pressão $p=0$, e um 
fluido de radiação é descrito $T^{\mu \nu}=p\left(U^{\mu} U^{\nu}+g^{\mu \nu}\right)$ neste caso temos equação de estado $p=3 \rho$. Definimos então o fator bariotrópico

$$
\omega=\frac{p}{\rho}
$$

onde esse fator constante assume valores de acordo com o tipo de matéria 5 .

$\begin{array}{ll}\text { matéria } & \omega \\ \text { ordinária } & 0 \\ \text { radiação } & \frac{1}{3} \\ \text { curvatura } & -\frac{1}{3} \\ \text { vacuo } & -1\end{array}$

Nenhuma imposição sobre o tensor de momento-energia é feita a priori 7 14]. Alguns fluidos são repulsivos, outros podem violar causalidade, ou serem ultra-relativisticos essas condições de energia classificam os fluidos quanto a estes critérios que vamos abordar a seguir (Figura 1) Seja um dado vetor tipo tempo $t^{\mu}=\gamma(1, a, b, c), \gamma=$ $\frac{1}{1-a^{2}-b^{2}-c^{2}}$, com $g_{\alpha \beta} t^{\alpha} t^{\beta}$ portanto $a^{2}+b^{2}+c^{2}<1$, tenha$\operatorname{mos} T_{\mu \nu} U^{\mu} U^{\nu} \geq 0$ e para um vetor nulo $l^{\mu}=\left(1, a^{\prime}, b^{\prime}, c^{\prime}\right), 1=$ $a^{\prime 2}+b^{\prime 2}+c^{\prime 2}$, temos $T_{\mu \nu} l^{\mu} l^{\nu} \geq 0$. Deduzimos disso que

$$
\rho=T^{\mu \nu} U_{\mu} U_{\nu}, T_{\mu \nu} l^{\mu} l^{\nu}=(\rho+p)\left(U_{\mu} l^{\mu}\right)^{2} .
$$

Isso implica $\rho \geq 0$ e $(\rho+p) \geq 0$. Surge então a chamada condição de energia Fraca

\subsection{Condição de energia fraca}

Vamos analisar a condição de eneria fraca

$$
T_{\mu \nu} t^{\mu} t^{\nu}=\left(\rho+a^{2} p+b^{2} p+c^{2} p\right) \geq 0,
$$

se fazemos $a=b=c=0$ temos $\rho \geq 0$, alternativamente fazemos duas das constantes nulas, por exemplo $b=c=0$ e $a=1$ temos $(\rho+p) \geq 0$. Escrevemos então

$$
\rho \geq 0 ;(\rho+p) \geq 0
$$

Podemos ainda fazer $\frac{\rho+p}{\rho}=1+\frac{p}{\rho}$, fluidos barotrópicos são conhecidos pela equação de estado $p=\omega \rho$, então reescrevemos a condição de energia fraca

$$
\omega \geq-1
$$

Essa condição esta associada a causalidade do escoamento do fluido. Portanto o fluido escoa respeitando o cone de luz 12. Existe uma versão mais fraca dessa condição de energia, que passamos abordar agora

\subsection{Condição de energia nula}

Procedendo da mesma forma que procedemos para a condição de energia fraca 10 12, porém usamos vetores tipo luz, assim

$$
T_{\mu \nu} l^{\mu} l^{\nu} \geq 0
$$

onde $T_{\mu \nu} l^{\mu} l^{\nu}=\rho+a^{\prime 2} p+b^{\prime 2} p+c^{\prime 2} p \geq 0$, se fizermos $a^{\prime 2}+b^{\prime 2}+c^{\prime 2}=1$ então

$$
\rho+p \geq 0
$$

essa condição admite densidade negativa $\rho<0$, em casos de fluidos ultra-relativisticos, por isso $l^{\mu} l_{\mu}=0$ Usamos vetores tipo luz.

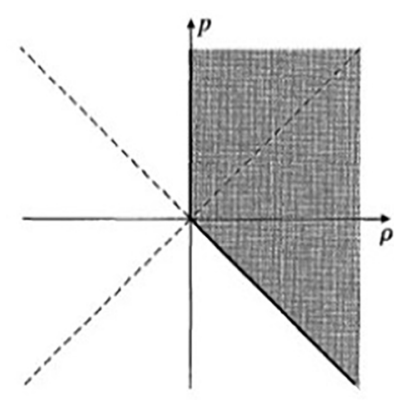

(a) WEC

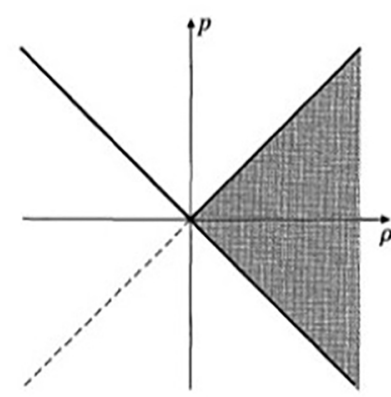

(d) NDEC

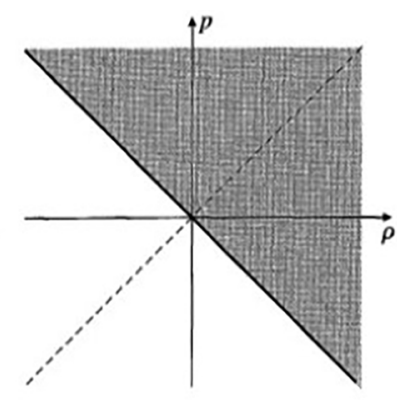

(b) NEC

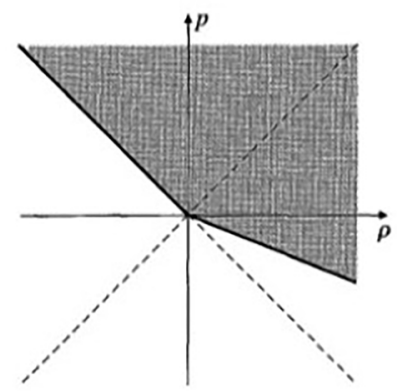

(e) SEC

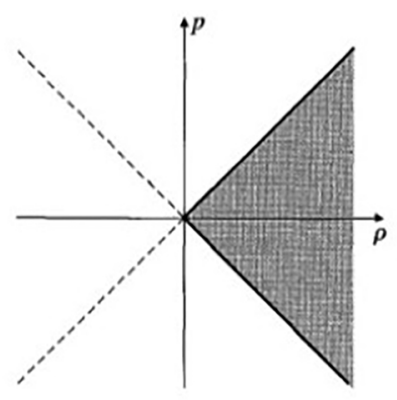

(c) DEC

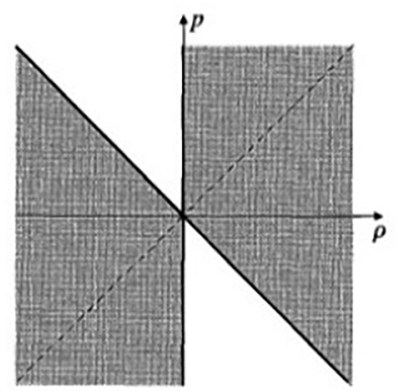

(f) $w \geqslant-1$

Figura 1: As condições de energia são aplicadas a fluidos perfeitos. Representando valores possiveis de densidade de energia e pressão. 5 


\subsection{Condição de energia dominante}

Um observador com quadri-velocidade $U^{\mu}$, vera uma quadri-corrente $-T_{\nu}^{\mu} U^{\nu}$, ou seja, temos que $-T_{\nu}^{\mu} U^{\nu}$ não pode ser tipo-espaço, que é equivalente a dizer $T_{\mu \nu} T_{\lambda}^{\nu} t^{\mu} t^{\lambda} \leq$ 0 então temos

$$
T_{\mu \nu} t^{\mu} t^{\nu} \geq 0 ; T_{\mu \nu} T_{\lambda}^{\nu} t^{\mu} t^{\lambda} \leq 0 .
$$

De $T_{\mu \nu} t^{\mu} t^{\nu} \geq 0$, obtemos $\rho \geq 0$. A quadri-corrente não ser um vetor tipo-espaço, o que implica que $\gamma^{2}\left(-\rho^{2}+\left(a^{2}+b^{2}+c^{2}\right) p^{2}\right)$, se fizermos $b=c=0$, ficamos com $\rho^{2} \geq a^{2} p^{2}$ e $a<1$, isso implica que $\rho \geq|p|$. Escrevemos finalmente

$$
\rho \geq 0 ; \rho \geq|p|
$$

As condições de energia acima não são as únicas, vamos enunciar mais duas condições de energia, que são associadas à presença de campo gravitacional. Faremos isso depois de algumas considerações sobre o tensor de momento-energia em espaços curvos

\section{Tensor de momento-energia como fonte para o campo gravitacional}

A ação dos campos gravitacionais em relatividade geral é associada à curvatura do espaço-tempo, estes espaçostempos são associadas à variedades riemaninas [5]. A curvatura dessas variedades é medida pelo tensor de Riemaann que definimos a seguir

$$
R_{\mu \nu \kappa}^{\lambda}=\partial_{\nu} \Gamma_{\mu \kappa}^{\lambda}-\partial_{\kappa} \Gamma_{\mu \nu}^{\lambda}-\Gamma_{\sigma \kappa}^{\lambda} \Gamma_{\mu \nu}^{\sigma}+\Gamma_{\sigma \nu}^{\lambda} \Gamma_{\mu \kappa}^{\sigma},
$$

temos que a conexão define a variedade sob a qual estamos trabalhando, no caso os simbolos de Christoffel

$$
\Gamma_{\mu \nu}^{\lambda}=\frac{1}{2} g^{\lambda \sigma}\left(\partial_{\mu} g_{\nu \sigma}+\partial_{\nu} g_{\sigma \mu}-\partial_{\sigma} g_{\mu \nu}\right)
$$

O tensor de Ricci é encontrado fazendo a contração $\lambda=\nu$, ou seja

$$
R_{\mu \kappa}=R_{\mu \nu \kappa}^{\nu}
$$

O escalar de Ricci é então

$$
R=g^{\mu \kappa} R_{\mu \kappa},
$$

esses objetos caracterizam a curvatura do espaço-tempo. Podemos agora definir a ação de Hilbert-Einstein 5

$$
S_{H}=\int R \sqrt{-g} d^{4} x
$$

usando o acoplamento minimo com uma ação de matéria $S_{m}$, temos

$$
\delta S=\delta S_{H}+8 \pi \delta S_{M}=0,
$$

onde $G=c=1$ ou seja

$$
\begin{gathered}
\delta S=\int\left(\frac{\delta(\sqrt{-g} R)}{\delta g^{\mu \nu}}+\frac{8 \pi}{\sqrt{-g}} \frac{\delta\left(\sqrt{-g} L_{M}\right)}{\delta g^{\mu \nu}}\right. \\
\left.\left.+\frac{8 \pi}{\sqrt{-g}} \frac{\partial\left(\sqrt{g} L_{M}\right)}{\partial\left(\partial_{k} g^{k \nu}\right)} \delta\left(\partial_{k} g^{k \mu}\right)\right)\right) \delta g^{\mu \nu} d^{4} x=0
\end{gathered}
$$

sendo $\mathcal{L}_{M}=\sqrt{-g} L_{M}$ a densidade de lagrangiana de matéria. Rearranjando os termos, temos

$$
\begin{aligned}
& \left.\delta S=\int\left(\frac{\delta \sqrt{-g}}{\delta g^{\mu \nu}} \frac{R}{2 \sqrt{-g}}-\frac{\delta R}{\delta g^{\mu \nu}}+\frac{8 \pi}{\sqrt{-g}} \frac{\delta\left(\mathcal{L}_{M}\right)}{\delta g^{\mu \nu}}\right)\right) \\
& \times \sqrt{-g} \delta g^{\mu \nu} d^{4} x
\end{aligned}
$$

chamamos o tensor de momento-energia

$$
T^{\mu \nu}=\frac{1}{\sqrt{-g}} \frac{\delta\left(\mathcal{L}_{M}\right)}{\delta g^{\mu \nu}},
$$

onde $R_{\mu \nu}=\frac{\delta R}{\delta g^{\mu \nu}}$, chegamos então a equação de Einstein

$$
R^{\mu \nu}-\frac{R}{2} g^{\mu \nu}-8 \pi T^{\mu \nu}=0,
$$

escrevendo o tensor de Einstein $G^{\mu \nu}=R^{\mu \nu}-\frac{R}{2} g^{\mu \nu}$, temos

$$
G^{\mu \nu}=8 \pi T^{\mu \nu}
$$

$\operatorname{com} G=c=1$

Pela idêntidade de Bianchi temos [5]:

$$
g^{\nu \sigma} g^{\mu \lambda}\left(\nabla_{\lambda} R_{\rho \sigma \mu \nu}+\nabla_{\rho} R_{\sigma \lambda \mu \nu}+\nabla \sigma R_{\lambda \rho \mu \nu}\right)=0
$$

se contrairmos essa expressão, temos

$$
\nabla^{\mu} R_{\rho \mu}-\nabla_{\rho} R+\nabla^{\mu} R_{\rho \mu}=0
$$

ou seja,

$$
\nabla^{\mu} R_{\rho \mu}=\frac{1}{2} \nabla_{\rho} R
$$

assim chegamos a a conservação do tensor de momentoenergia

$$
\nabla_{\mu} G^{\mu \nu}=\nabla_{\mu}\left[R^{\mu \nu}-\frac{R}{2} g^{\mu \nu}\right]=8 \pi \nabla_{\mu} T^{\mu \nu}=0 .
$$

A conservação do tensor de momento-energia gera a chamada equação de Tolemam-Opennhaimer-Volkov.

Podemos agora estudar duas condições de energia, associadas a regimes gravitacionais.

\subsection{Condição de energia nula dominante}

Consideremos agora duas condições de energia

$$
T_{\mu \nu} l^{\mu} l^{\nu} \geq 0 ; T_{\mu \nu} T_{\lambda}^{\nu} l^{\mu} l^{\lambda} \leq 0,
$$

a primeira $T_{\mu \nu} l^{\mu} l^{\nu}$, ja foi mencionada e implica $\rho+p \geq 0$, por sua vez $T_{\mu \nu} T_{\lambda}^{\nu} l^{\mu} l^{\lambda}=\rho^{2}+\left(a^{\prime 2}+b^{\prime 2}+c^{\prime 2}\right) p$. Sabemos que $a^{\prime 2}+b^{\prime 2}+c^{\prime 2}=1, \operatorname{logo} \rho+p \leq 0$. Logicamente a única condição restante é $p=-\rho$. Essa condição de energia exclui todas as fontes, excluidas pela Condição de energia dominante, exceto a energia do vácuo 12

$$
p=-\rho .
$$

Da equação 49, vemos que $T^{\mu \nu}=p g^{\mu \nu}$ A condição de energia nula está associada à constante cosmológica. A solução da equação de Einstein para este tipo de tensor de momento-energia, implica em um espaço-tempo dito maximal $R_{\mu \nu} \propto g_{\mu \nu}, 5,10,12$. 


\subsection{Condição de energia forte}

Essa condição está ligada à gravidade atrativa, usando a equação de Einstein $R_{\mu \nu}=\left(T_{\mu \nu}-\frac{1}{2} T g_{\mu \nu}\right)$, temos

$$
R_{\mu \nu} t^{\mu} t^{\nu}=\left(T_{\mu \nu}-\frac{1}{2} T g_{\mu \nu}\right) t^{\mu} t^{\nu} \geq 0
$$

$T_{\mu \nu} t^{\mu} t^{\nu} \geq 0$ implica $\rho+p \geq 0$, já $\frac{1}{2} T g_{\mu \nu} t^{\mu} t^{\nu}=-\frac{1}{2}(\rho-3 p)$ pela equação 51. Entao $R_{\mu \nu} t^{\mu} t^{\nu}=\left(T_{\mu \nu}-\frac{1}{2} T g_{\mu \nu}\right) t^{\mu} t^{\nu}=$ $\rho+p+\frac{1}{2}(\rho-3 p) \geq 0$, como $\rho+p \geq 0$ so nos resta escrever que $\rho-3 p \geq 0$. Escrevemos finalmente

$$
\rho+p \geq 0 ; \rho-3 p \geq 0 \text {. }
$$

A violação dessa condição gera gravidade repulsiva.

\subsection{Demonstração da TOV isotrópica: fluido ideal em uma métrica genérica}

Partimos da equação 49 e derivamos $\nabla_{\mu} T^{\mu \nu}$, então

$$
\begin{aligned}
& \nabla_{\mu} T^{\mu \nu}=\nabla_{\mu}(p+\rho) U^{\mu} U^{\nu}+(\rho+p)\left(\left(\nabla_{\mu} U^{\mu}\right) U^{\nu}\right. \\
& \left.+U^{\nu} \nabla_{\mu} U^{\mu}\right)+\nabla_{\mu} p g^{\mu \nu} .
\end{aligned}
$$

A equação da continuidade, ou de conservação de massa é $\nabla_{\mu}\left(\rho U^{\mu}\right)=U^{\mu} \nabla_{\mu} \rho+(\rho+p) \nabla_{\mu} U^{\mu}=0$, vemos então que

$$
\nabla_{\mu} T^{\mu \nu}=\left(\nabla_{\mu} p\right) U^{\mu} U^{\nu}+(\rho+p) U^{\nu} \nabla_{\mu} U^{\mu}+\left(\nabla_{\mu} p\right) g^{\mu \nu} .
$$

Sabemos que $\nabla_{\mu} U^{\nu}=\partial_{\mu} U^{\nu}+\Gamma_{\alpha \mu}^{\nu} U^{\alpha}$, e que temos quadri-velocidade $U^{t}=\sqrt{g^{00}}, U^{1}=0$. Portanto a única componente diferente de zero da derivada da 4 -velocudade é $\nabla_{r} U^{t}=\partial_{r}\left(\sqrt{g^{t t}}\right)+\Gamma_{t r}^{t} \sqrt{g^{t t}}$, escrevemos pois

$$
\left(\nabla_{\mu} p\right) g^{00}+(\rho+p) \sqrt{g^{00}} \nabla_{\mu} \sqrt{g^{00}}+\left(\nabla_{\mu} p\right) g^{00}=0,
$$

contraindo com a métrica

$$
2 \nabla_{\mu} p+(\rho+p) \sqrt{g^{00}} \Gamma_{00}^{\nu} g^{t t} g_{\mu \nu} g^{00}=0,
$$

finalmente

$$
\nabla_{\mu} p=-(\rho+p) \sqrt{g^{00}} \Gamma_{00}^{\nu} g^{00} g_{\mu \nu}\left(g^{00}\right)^{-1}
$$

chegamos então a equação que representa a conservação da massa-energia

$$
\nabla_{\mu} p=-(\rho+p) \sqrt{g^{00}} \Gamma_{00}^{\nu} g_{\mu \nu}
$$

\subsection{Demonstração da TOV Isotrópica: métrica de um objeto esférico}

Vamos estudar a equação de Tolemam-Openhaimer-Volkov (TOV) para uma dada distribuição de massa esférica. A TOV é a equação que corresponde ao equilibrio hidroestático de um fluido relativístico. Podemos também interpretar a TOV como a conservação do tensor de momento-energia [16]. A métrica de uma distribuição esférica de massa $m$

$$
\begin{aligned}
& d s^{2}=-e^{\Phi(r)} d t^{2}+\left(1-\frac{2 m}{r}\right)^{-1} d r^{2} \\
& +r^{2}\left(d \theta^{2}+\sin (\theta) d \phi^{2}\right)
\end{aligned}
$$

sendo a métrica dada em forma matricial

$$
g^{\mu \nu}=\left[\begin{array}{rrcc}
-e^{\Phi(r)} & 0 & 0 & 0 \\
0 & \left(1-\frac{2 m}{r}\right)^{-1} & 0 & 0 \\
0 & 0 & r^{2} & 0 \\
0 & 0 & 0 & r^{2} \sin (\theta)
\end{array}\right] .
$$

Podemos então calcular os símbolos de Christoffel associados à métrica de uma distribuição esférica

$$
\begin{gathered}
\Gamma_{t r}^{t}=\Phi^{\prime} \\
\Gamma_{t t}^{r}=\Phi^{\prime} e^{2 \Phi}\left(1-\frac{2 m}{r}\right), \\
, \Gamma_{r r}^{r}=\frac{r m^{\prime}-m}{r^{2}-2 m r}, \\
\Gamma_{r \theta}^{\theta}=\Gamma_{r \phi}^{\phi}=\frac{1}{r} \\
\Gamma_{\theta \phi}^{\phi}=-\sin (\theta) \cos (\theta), \\
\Gamma_{\theta \theta}^{r}=\Gamma_{\phi \phi}^{\theta}=\csc ^{2}(\theta), \\
\Gamma_{\phi \phi}^{r}=2 m-r .
\end{gathered}
$$

Aqui é preciso dizer que estamos usando um sistema de unidades em que massa e raio tem a mesma unidade. O tensor de Ricci associado a essas conexões têm as seguintes componentes

$$
\begin{aligned}
& R_{t t}=e^{2 \Phi}\left[\left(\Phi^{\prime \prime}+\Phi^{\prime 2}\right)\left(1-\frac{2 m}{r}\right)\right. \\
& \left.+\Phi^{\prime}\left(\frac{2 r-3 m-r m^{\prime}}{r^{2}}\right)\right]
\end{aligned}
$$

$$
\begin{gathered}
R_{r r}=\left(1-\frac{2 m}{r}\right)^{-1}\left[\frac{\left(r m^{\prime}-m\right)\left(2+r m^{\prime}\right)}{r^{3}}\right] \\
-\Phi^{\prime \prime}-\Phi^{\prime 2} \\
R_{\theta \theta}=\csc ^{2}(\theta) R_{\phi \phi}=(2 m-r) \Phi^{\prime}+m^{\prime}+\frac{m}{r} .
\end{gathered}
$$

. O escalar de Ricci é

$$
\begin{aligned}
& R=g^{\mu \nu} R_{\mu \nu}=2\left[\frac{2 m^{\prime}}{r}+\Phi^{\prime}\left(3 m-2 r+r m^{\prime}\right)\right. \\
& \left.-\left(1-\frac{2 m}{r}\right)\left(\Phi^{\prime \prime}+\Phi^{\prime 2}\right)\right] .
\end{aligned}
$$

Usando a componente $r r$ da equação de Einstein que se lê

$$
G_{r r}=\frac{2}{r}\left(\Phi^{\prime}-\frac{m}{1-\frac{2 m}{r}}\right)=\frac{8 \pi p}{1-\frac{2 m}{r}},
$$


achamos então

$$
\Phi^{\prime}=\frac{m+4 \pi r^{3} p}{r(r-2 m)} .
$$

A componente $t t$ é

$$
G_{t t}=\frac{2 m^{\prime} e^{2 \Phi}}{r^{2}}=8 \pi \rho e^{2 \Phi}
$$

onde $m^{\prime}=\frac{d m}{d r}=4 \pi \rho r^{2}$, é a equação da continuidade . Podemos escrever o tensor de momento-energia

$$
T^{\mu \nu}=\left[\begin{array}{rrcc}
\rho e^{-2 \Phi} & 0 & 0 & 0 \\
0 & \left(1-\frac{2 m}{r}\right) p & 0 & 0 \\
0 & 0 & \frac{p}{r^{2}} & 0 \\
0 & 0 & 0 & p \frac{\csc ^{2}(\theta)}{r^{2}}
\end{array}\right] .
$$

Dado que as funções são dependentes da coordenada $r$, temos a derivada covariante do tensor de momentoenergia.

$$
\begin{gathered}
\nabla_{r} T^{r \nu}=\partial_{r} T^{r \nu}+T^{\sigma \mu} \Gamma_{\sigma \mu}^{r}+T^{r \sigma} \Gamma_{\sigma \nu}^{\nu} \\
\partial_{r} T^{r r}+T^{r r}\left(\Gamma_{r \nu}^{\nu}+\Gamma_{r r}^{r}\right)+T^{\theta \theta} \Gamma_{\theta \theta}^{r}+T^{\phi \phi} \Gamma_{\phi \phi}^{r} \\
=\left(1-\frac{2 m}{r}\right)\left[\frac{d p}{d r}+(\rho+p) \Phi^{\prime}\right]=0,
\end{gathered}
$$

assim a TOV

$$
\frac{d p}{d r}=-(\rho+p) \frac{m+4 \pi p r^{3}}{r(r-2 m)},
$$

junto com a equação da continuidade ,

$$
\frac{d m}{d r}=4 \pi \rho r^{2}
$$

formam as chamadas equações de estrutura para uma estrela 16.

\section{Fluido anisotrópico}

Definindo o tensor de momento-energia de um gás ideal anisotrópico 9,13

$$
T^{\mu \nu}=\left(\rho+p_{t}\right) U^{\mu} U^{\nu}+p g^{\mu \nu}+\left(p_{t}-p\right) s^{\mu} s^{\nu}
$$

onde $s^{\mu}$ é perpendicular a quadri-velocidade de escoamento do fluido $U^{\mu}=(1,0,0,0), s^{\mu} U_{\mu}=0$, Em termos matriciais

$$
T^{\mu \nu}=\left[\begin{array}{cccc}
\rho & 0 & 0 & 0 \\
0 & p & 0 & 0 \\
0 & 0 & p_{t} & 0 \\
0 & 0 & 0 & p_{t}
\end{array}\right]
$$

O Elemento

$$
\Delta=p_{t}-p
$$

é o chamado fator anisotrópico, $\Delta>0, p_{t}>p$, ou seja, um fator repulsivo, ou $\Delta<0, p_{t}<p$, neste caso a anisotropia colabora com a ação gravitacional $\mathrm{O}$ traço do tensor de momento-energia é

$$
T=\rho-p-2 p_{t}
$$

Vamos agora estudar as limitações conhecidas como condições de energia [?,13]. Vamos gerar condições de energia semelhantes as condições para o caso isotrópico, semellhantes no sentido que os vetores tipo-tempo e tipoluz, serão escolhidos para se adequarem ao tensor de momento-energia com componentes diferentes.

\subsection{Condição de energia fraca}

Como nas seções anteriores a condição de energia fraca é

$$
T_{\mu \nu} t^{\mu} t^{\nu} \geq 0
$$

sendo $t^{\mu}$ vetor tipo-tempo, estabelecemos que $t^{\mu}=(1, a, b, c)$, então $T_{\mu \nu} t^{\mu} t^{\nu}=\rho+a p+b p_{t}+c p_{t}$. Escolhendo $a=b=$ $c=1$ chegamos à positividade do traço

$$
\rho+p+2 p_{t} \geq 0
$$

Aqui o fator barotrópico fica modificado $1+\omega+2 \frac{p_{t}}{\rho} \geq 0$

$$
\omega \geq-1-2 \frac{p_{t}}{\rho}
$$

a causalidade fica então modificada, passando a levar em conta a pressão tangencial.

\section{2. condição de energia nula}

Usando os vetores nulos $l^{\mu=1 \cdot a^{\prime}, b^{\prime}, c^{\prime}}$ a condição de energia nula é

$$
T_{\mu \nu} l^{\mu} l^{\nu} \geq 0
$$

Verificamos pois que, $T_{\mu \nu} l^{\mu} l^{\nu}=\rho+a^{\prime 2} p+\left(b^{\prime 2}+c^{\prime 2}\right) p_{t}$, escolhendo $b^{\prime}=c^{\prime}=0$, obrigatóriamente temos $a^{\prime}=1$, ficamos com $\rho+p \geq 0$, alternativamente fazemos $a^{\prime}=0$ ficamos com $\rho+p_{t} \geq 0$, então

$$
\rho+p \geq 0, \rho+p_{t} \geq 0
$$

Ficamos com duas inequações semlhantes, que represetam condições para fluidos ultra-relativísticos.

\section{3. condição de energia dominante}

A condição de energia dominante é

$$
T_{\mu \nu} t^{\mu} t^{\nu} \geq 0, T_{\mu \nu} T_{\lambda}^{\nu} t^{\mu} t^{\lambda} \leq 0
$$

De $T_{\mu \nu} t^{\mu} t^{\nu} \geq 0$, obtemos $\rho \geq 0$. A quadricorrente não ser um vetor tipo-espaço implica que $\gamma^{2}\left(-\rho^{2}+\left(a^{2} p^{2}+\left(b^{2}+c^{2}\right) p_{t}\right)\right)$, se fizermos $b=c=0$, ficamos com $\rho^{2} \geq a^{2} p^{2}$ e $a<1$, implica que $\rho \geq|p|$. Seguindo um raciocionio similar $a=0$, chegamos a $\rho \geq\left|p_{t}\right|$. Escrevemos finalmente

$$
\rho \geq 0 ; \rho \geq|p| ; \rho \geq\left|p_{t}\right| .
$$




\section{4. condição de energia nula-dominante}

A condição de energia nula dominante é expressa

$$
T_{\mu \nu} l^{\mu} l^{\nu} \geq 0 ; T_{\mu \nu} T_{\lambda}^{\nu} l^{\mu} l^{\nu} \leq 0
$$

$T_{\mu \nu} l^{\mu} l^{\nu}$, já foi calculada e implica $\rho+p \geq 0, \rho+p_{t} \geq$ 0 , por sua vez $T_{\mu \nu} T_{\lambda}^{\nu} l^{\mu} l^{\nu}=\rho^{2}+a^{\prime 2} p+\left(b^{\prime 2}+c^{\prime 2}\right) p_{t}$. Sabemos que $a^{\prime 2}+b^{\prime 2}+c^{\prime 2}=1$,fazendo $b^{\prime}=c^{\prime}=0$ $\operatorname{logo} \rho+p \leq 0$. Logicamente a única condição restante é $p=-\rho$. Podemos ainda fazer $a^{\prime}=0$, implicando que $\rho+p_{t} \leq 0$. Portanto temos tambem $p_{t}=-\rho$, logo a energia do vácuo não é anisotrópica. Resumindo

$$
p_{t}=p=-\rho
$$

expressa os resultados.

\section{5. condição de energia forte}

Essa condição esta ligada à gravidade atrativa, usando a equação de Einstein temos $R_{\mu \nu}=\left(T_{\mu \nu}-\frac{1}{2} T g_{\mu \nu}\right)$

$$
R_{\mu \nu} t^{\mu} t^{\nu}=\left(T_{\mu \nu}-\frac{1}{2} T g_{\mu \nu}\right) t^{\mu} t^{\nu} \geq 0,
$$

novamente $T_{\mu \nu} t^{\mu} t^{\nu} \geq 0$, implica $\rho-p-2 p_{t} \geq 0$, já $\frac{1}{2} T g_{\mu \nu} t^{\mu} t^{\nu}=-\frac{1}{2}\left(\rho-p-2 p_{t}\right)$ pela equação 112$)$. Entao $R_{\mu \nu} t^{\mu} t^{\nu}=\left(T_{\mu \nu}-\frac{1}{2} T g_{\mu \nu}\right) t^{\mu} t^{\nu}=\rho+p+2 p_{t}+\frac{1}{2}(\rho-p-$ $\left.2 p_{t}\right) \geq 0$, como $\rho+p+2 p_{t} \geq 0$ só nos resta escrever que $\rho-p-2 p_{t} \geq 0$. Escrevemos finalmente

$$
\rho+p+2 p_{t} \geq 0 ; \rho-p-2 p_{t} \geq 0 .
$$

A violação dessa condição gera gravidade repulsiva.

\subsection{Demonstração da TOV anisiotrópica}

A exemplo do que fizemos no caso isotrópico, escrevemos aqui a métrica em termos de funções métricas $\nu(r), \lambda(r)$ 9 .

$$
d s^{2}=-e^{\nu(r)} d t^{2}+e^{\lambda(r)} d r^{2}+r^{2}\left(d \theta^{2}+\sin ^{2}(\theta) d \phi^{2}\right),
$$

podemos agora usar a equação de Einstein 778 . A componente $t t$ é

$$
e^{-\lambda(r)}\left(\frac{\lambda^{\prime}}{r}-\frac{1}{r^{2}}\right)+\frac{1}{r^{2}}=8 \pi \rho
$$

e a componente $r r$

$$
e^{-\lambda(r)}\left(\frac{1}{r^{2}}+\frac{\nu^{\prime}}{r}\right)-\frac{1}{r^{2}}=8 \pi p,
$$

a componentes angular $\theta \theta$ gera

$$
\frac{1}{2}\left(\frac{1}{2}\left(\nu^{\prime}\right)^{2}+\nu^{\prime \prime}-\frac{1}{2} \lambda^{\prime} \nu^{\prime}+\frac{1}{r}\left(\nu^{\prime}-\lambda^{\prime}\right)\right)=8 \pi p_{t} .
$$

A equação para $p_{t}$ representa a pressão anisotrópica, aqui se manifesta a diferença com o caso isotrópico . Esse é o modelo usual para estrelas estranhas anisotrópicas.
Consideremos o equilibrio hidroestático 20]

$$
F_{g}+F_{\text {Hidro }}+F_{\text {anisio }}=0,
$$

onde $F_{g}=\frac{1}{2}(\rho+p) d_{r} \nu(r)$ é a força gravitacional, $F_{\text {hidro }}=$ $-\frac{d p}{d r}$ a força hidroestática e a força anisotrópica $F_{a n i}=$ $2 \frac{\left(p_{t}-p\right)}{r}$. Podemos então escrever a TOV na sua versão anisotrópica como

$$
\frac{d p}{d r}=-m \frac{\rho+p}{r^{2}} \frac{d \nu(r)}{d r}+\frac{2}{r}\left(p_{t}-p\right)
$$

A primeira parcela da TOV é idêntica ao caso isotrópico, pois as equações 102 são muito similtares a equação 126). $\nu^{\prime}(r)=\Phi^{\prime}(r)$. Temos escrevemos finalmente a TOV. Assim como

$$
\frac{d p}{d r}=-(\rho+p) \frac{m+4 \pi p r^{3}}{r(r-2 m)}+\frac{2}{r}\left(p_{t}-p\right)
$$

que é a TOV isotrópica .

\section{A constante cosmológica}

Também é possível construirmos um tensor de momentoenergia levando em conta a interação da matéria com a constante cosmológica. Neste caso a equação de Einstein é modificada para

$$
R_{\mu \nu}-\frac{R}{2} g_{\mu \nu}+\Lambda g_{\mu \nu}=8 \pi T_{\mu \nu}
$$

a versão homogênea desta equação seria a equação de Einstein para o vácuo, conhecida como equação de De Sitter [5, 10-12,21,

$$
R_{\mu \nu}-\frac{R}{2} g_{\mu \nu}+\Lambda g_{\mu \nu}=0
$$

tem como solução

$$
R_{\mu \nu}=\Lambda g_{\mu \nu}
$$

um tensor de Ricci proporcional a métrica, poderiamos alternativamente pensar em um tensor energia momento proporcional a métrica $T_{\mu \nu}=\Lambda g_{\mu \nu}$, essa condição, é associada a métrica de De Sitter

$d s^{2}=-\left(1-\frac{\Lambda r^{2}}{3}\right) d t^{2}+\frac{1}{1-\frac{\Lambda r^{2}}{3}} d l^{2}+r^{2}\left(d \theta^{2}+\sin \theta d \phi^{2}\right)$

e o tensor energia momento proporcional a métrica, implica em uma equação de estado 49

$$
p=-\rho
$$

[10,11, tendo um fator barimétrico $\omega=-1$, uma equação de estado deste tipo, obedece a condição de energia NDEC

Para efetivamente calcularmos a TOV precisamos de uma métrica 21] assim usamo

$$
d s^{2}=e^{\nu} d t^{2}-e^{\lambda(r)} d r^{2}-r^{2}\left(d \theta^{2}+\sin ^{2} \theta d \phi^{2}\right),
$$


explicitando as componentes da métrica $g_{t t}=e^{\nu}, g_{r r}=$ $-e^{\lambda}, g_{\theta \theta}=-r^{2}, g_{\phi \phi}=-r^{2} \sin ^{2}(\theta)$ e usando a relação

$$
g^{\mu \nu} g_{\nu \lambda}=\delta_{\lambda}^{\mu}
$$

escrevemos então a métrica na forma matricial

$$
g^{\mu \nu}=\left[\begin{array}{rrcc}
e^{-\nu} & 0 & 0 & 0 \\
0 & -e^{\lambda} & 0 & 0 \\
0 & 0 & -\frac{1}{r^{2}} & 0 \\
0 & 0 & 0 & \frac{1}{(r \sin \theta)^{2}}
\end{array}\right],
$$

aqui as funções métricas $\nu=\nu(r)$ e $\lambda=\lambda(r)$. Calculamos o tensor de Ricci $R_{\mu \nu}=\partial_{\alpha} \Gamma_{\mu \nu}^{\alpha}-\partial_{\nu} \Gamma_{\mu \alpha}^{\alpha}+\Gamma_{\beta \alpha}^{\alpha} \Gamma_{\mu \nu}^{\beta}-$ $\Gamma_{\beta \mu}^{\alpha} \Gamma_{\alpha \nu}^{\beta}$, lembrando sempre que $R_{\beta}^{\alpha}=g^{\alpha \mu} R_{\mu \beta}$. Escrevemos as componentes da equação de Einstein.

Componente $t$ :

$$
\begin{gathered}
G_{t}^{t}=-8 \pi T^{t}{ }_{t} \\
8 \pi \rho=e^{-\lambda}\left(\frac{\lambda^{\prime}}{r}-\frac{1}{r^{2}}\right)+\frac{1}{r^{2}}-\Lambda,
\end{gathered}
$$

Componente $r r$ :

$$
\begin{gathered}
G_{r}^{r}=8 \pi T_{r}^{r}, \\
8 \pi p=e^{-\lambda}\left(\frac{\nu^{\prime}}{r}+\frac{1}{r^{2}}\right)-\frac{1}{r^{2}}+\Lambda
\end{gathered}
$$

para uma estrela estática consideramos

$$
\frac{d \rho}{d t}=\frac{d p}{d t}=0
$$

Assim a derivada covariante do tensor de momentoenergia que induz o seguinte resultado

$$
(\rho+p)\left(\partial_{\mu} u_{\sigma}\right) u^{\mu}+\partial_{\sigma} p+\partial_{\mu} p u^{\mu} u_{\sigma}=0 .
$$

Lembramos a componente $r r$ da métrica $g_{r r}=-e^{\lambda}$, então

$$
(\rho+p)\left(\partial_{\mu} u_{t}\right) u^{\mu}-\partial_{r} p=0,
$$

assim

$$
\partial_{r} p=-\frac{(\rho+p)}{2} \frac{d \nu}{d r}
$$

Definimos agora a massa para uma casca esférica de raio $r$ como

$$
m(r)=4 \pi \int_{0}^{r} \rho\left(r^{\prime}\right) r^{\prime 2} d r^{\prime}
$$

diferenciando a massa e substituindo na componente temporal da equação de Einstein (140) obtemos

$$
\frac{d m}{d r}=-\frac{1}{2} \frac{d}{d r}\left[e^{-\lambda} r-r+\frac{\Lambda r^{3}}{3}\right],
$$

integrando temos

$$
2 \int_{0}^{r} \frac{d m}{d r} d r=-\int_{0}^{r} \frac{1}{2} \frac{d}{d r}\left[e^{-\lambda} r-r+\frac{\Lambda r^{3}}{3}\right] d r
$$

assim chegamos finalmente que a componente $r r$ da métrica

$$
e^{-\lambda(r)}=1-\frac{2 m(r)}{r}-\frac{\Lambda r^{3}}{3},
$$

somando as componentes radial e temporal da equação de Einstein, respectivamente as equações (140, 1 (142), temos

$$
8 \pi(\rho+p)=\frac{\lambda^{\prime} e^{-\lambda}}{r}+\frac{e^{-\lambda} \nu^{\prime}}{r} .
$$

Derivando a equação 150 com respeito a coordenada radial temos

$$
\lambda^{\prime} e^{-\lambda}=\frac{2 m^{\prime}}{r}-\frac{2 m}{r^{2}}+\frac{2 \Lambda r}{3}
$$

substituimos agora as equações 152 , 150 na equação (151) obtemos

$8 \pi(\rho+p)=\left(\frac{2 m^{\prime}}{r}-\frac{2 m}{r^{3}}+\frac{2 \Lambda}{3}\right)+\frac{1}{r} \frac{d \nu}{d r}\left(1-\frac{2 m}{r}-\frac{\Lambda r^{2}}{3}\right)$

substituindo (146) na equação 153 chegamos finalmente a TOV com constante cosmologica:

$$
\frac{d p}{d r}=-\rho\left(1+\frac{p}{\rho}\right) \frac{m+4 \pi p r^{3}-\frac{\Lambda r^{3}}{3}}{r^{2}\left(1-\frac{2 m}{r}-\frac{\Lambda r^{2}}{3}\right)} .
$$

A introdução da constante cosmológica gera um efeito interessante, se fizermos $m=0$, termos um efeito associado à pressão do vácuo

$$
\frac{d p}{d r}=-\rho\left(1+\frac{p}{\rho}\right) \frac{4 \pi p r^{3}-\frac{\Lambda r^{3}}{3}}{r^{2}\left(1-\frac{\Lambda r^{2}}{3}\right)} .
$$

Costumamos considerar que a equação de estado do vácuo é $p=-\rho$, obdecendo a condição de energia $(79)$, induziria a equação acima a ser 155 idênticamente nula, logo a pressão do vacuo fosse uma constante. Contudo se considerarmos o efeito do termo da constante cosmologica como uma pressão extra, de forma semelhante ao que fazemos na presença de pressão tangencial, poderiamos considerar que a parte associada à constante $(\Lambda>0)$ cosmológica fosse uma anisotropia. Uma pressão extra negativa, que tenta compensar os efeitos da pressão radial.

\section{Conclusão}

O estudo do tensor de momento-energia é um tópico fundamental em relatividade, a revisão das condições clássicas de energia é uma necessidade dado a sua relevância para diversas linhas de pesquisa em astrofísica e cosmologia. O surgimento de fluidos relativisticos escuros e exóticos, traz a necessidade de revisarmos a suas condições de energia em especial quanto a causalidade. A anisotropia do tensor de momento-energia é um tema que ainda suscita muitas duvidas entre os discentes e a constante cosmológica vem cada vez mais sendo associada a objetos ultra-densos. Revisamos estes temas 
calculando a conservação do tensor de momento-energia, buscamos contribuir com a discussão acerca das condições de energia, tanto no caso isotrópico como anisotrópico, identicando exatamente qual a condição de energia associada à energia do vácuo.

Pretendemos no futuro estender essa revisão a fluidos com simetria esférica e a fluidos exóticos, assim como o fenômeno da superfluides, que cada vez mais se torna importante tanto no estudo de matéria de quark, quanto no estudo de fluidos escuros.

\section{Referências}

[1] V. A. Bezerra, Revista Latino americana de filosofia e história da ciência 4, 177 (2006).

[2] G. A.ToBkaty, A history and philosophy of fluid mechanics (Dover Publications, New York, 1994).

[3] L. D. Landau and E.M. Lifschitz, The Classical Theory of Fields (Pergamon Press, Oxford, 1975).

[4] J. Frenel, Principios da Eletrodinâmica Clássica (EDUSP, São Paulo, 2005).

[5] S. Carroll, Spacetime and Geometry: An Introduction to General Relativity (Addison-Wesley Professional, Boston, 2004).

[6] S. W. Hawking and G.F.R. Ellis, The Large Scale Structure of Space-Time (Cambridge University Press, Cambridge, 1973).

[7] C. S. Santos, Condições de Energia de Hawking-Ellis $e$ as equações de Raychaudhury. Tese de Doutorado, Universidade Federal do Rio de Janeiro, Rio de Janeiro (2011).

[8] A. R. Liddle, arXiv:astro-ph/9901124v1 (1999).

[9] M. Kalam, F. Rahaman, S. Ray, S.K.M. Hossein, I. Karar and J.Naskar, arXiv:1201.5234 (2012).

[10] S. M. Carroll, Living Rev. Relativ. 4, 5 (2001).

[11] S. M. Carroll, arXiv:astro-ph/0107571 (2001).

[12] S. M. Carroll, M. Hoffman and M.Trodden, Phys.Rev. D 68, 023509 (2003).

[13] R. Chan, M.F.A. da Silva and J.F. Villas da Rocha, Modern Physics Letters A 24, 1137 (2009).

[14] F. M. Santos, Fluidos Ideias em Relatividade Geral e Cosmologia. Tese de Doutorado, Universidade Federal do Espírito Santo, Vitória (2016).

[15] F. S. Bemfica, M.M. Disconzi and J. Noronha, Phys. Rev. D 98, 104064 (2018).

[16] N. K. Glendenning, Compact Stars: Nuclear Physics, Particle Physics, and General Relativity (Springer Verlag, New York, 1997).

[17] H. Rodrigues, S.B. Duarte and J.C.T. Oliveira, The Astrophysical Journal 30, 1 (2011).

[18] G. E. Volovik, Phys.Rept. 351195 (2001).

[19] R. Dey, S. Liberati and R. Turcati, Phys. Rev. D 94, 104068 (2016).

[20] J. R. Oppenheimer and G.M. Volkoff, Phys. Rev. 55, 374 (1939).

[21] O. Zubairi, A. Romero and F. Weber, Journal of Physics: Conference Series 615 (2015). 